\title{
Indifference Prices of Structured Catastrophe (CAT) Bonds
}

\author{
Masahiko Egami * $\quad$ Virginia R. Young ${ }^{\dagger}$
}

17 August 2007

\begin{abstract}
We present a method for pricing structured CAT bonds based on utility indifference pricing. The CAT bond considered here is issued in two distinct notes called tranches, specifically senior and junior tranches each with its own payment schedule. Our contributions to the literature of CAT bond pricing are two-fold. First, we apply indifference pricing to structured CAT bonds. We find a price for the senior tranche as a relative indifference price, that is, relative to the price of the junior tranche. Alternatively, one could take the approach that the senior tranche is priced first and the price of the junior tranche is relative to that. Second, instead of simply supposing that the "not-issue-a-CAT-bond" strategy of the reinsurer is to do nothing, we suppose that the reinsurer reduces its risk by reinsuring proportionally less of the claims. We assume the reinsurance claims follow a (Poisson) jump-diffusion process.
\end{abstract}

Key Words: Catastrophe (CAT) bond, structured derivative security, indifference price, exponential utility, jump diffusion, reinsurance strategy

JEL Classification: G22, G13

\section{Introduction}

Catastrophe bonds (CAT bond, thereafter) are one of the most important insurance-linked financial securities. Investors purchase CAT bonds from the issuer, a special purpose vehicle that simultaneously enters into a reinsurance contract with a reinsurance company. The coupon and principal payments depend on the performance of a pool or index of natural catastrophe risks. The default of a CAT bond occurs when catastrophic events (such as earthquakes, hurricanes and floods) of some degree occurs. In a simple transaction, if the default occurs, no cash is paid to the investor thereafter. Otherwise, the coupons and principal are paid out to the buyer of the CAT bond. Due to the magnitude of losses caused by a large catastrophe, a CAT bond is an innovative financial instrument by which a reinsurance company transfers the risk of a possible large payment caused by catastrophic events to the capital market.

\footnotetext{
${ }^{*}$ Corresponding author. Tel:+81-75-753-3430; Fax:+81-75-753-3492. Department of Mathematics, University of Michigan, Ann Arbor, MI 48109, USA / Graduate School of Economics, Kyoto University, Kyoto, 606-8501, Japan. Email: egami@econ.kyoto-u.ac.jp.

${ }^{\dagger}$ Department of Mathematics, University of Michigan, Ann Arbor, MI 48109, USA, Email: vryoung@umich.edu.
} 
The recent paper by Lee and Yu [5] cites McGhee [6] regarding the size of the CAT bond market, namely a total issuance of $\$ 1.22$ billion in 2002 and $\$ 1.73$ billion in 2003 . According to Swiss Re, 1 "the market is growing: the volume of bonds outstanding has steadily increased since 1997 to a current level of approximately USD 3 billion." Due to the recent event of Hurricane Katrina in 2005, it can be reasonably assessed that the market size of a new issuance is around \$3-4 billion in recent years. To address diverse investors with different risk-return preferences, a structured CAT bond is usually issued in the market. In these transactions, CAT bonds are issued in separate notes (called "tranches") with different payment schedules and seniority. Senior tranches usually have investment grades from the rating agencies since the holders of senior tranches have higher priority of being paid. On the other hand, junior tranches have speculative grades, but the higher risk is compensated by larger coupon payments. See, for example, Shimpi [9] (page 179) for a transaction completed by Swiss Re.

In a broad sense, CAT bonds are categorized as catastrophe derivative securities. In the literature, there are several papers with respect to catastrophe derivatives. Geman and Yor [3] analyze catastrophe options with payoff $(L(T)-K)^{+}$where $L$ is the aggregate claim process modeled by a jump-diffusion process. Dassios and Jang [2] use a doubly-stochastic Poisson process for the claim process to price catastrophe reinsurance contract and derivatives. Jaimungal and Wang [4] study the pricing and hedging of catastrophe put options (CatEPut) under stochastic interest rates with a compound Poisson process. Among them, only few papers directly attempt to price CAT bonds: Cox and Pedersen [1] price a CAT bond under a term structure model together with an estimation of the probability of catastrophic events. Lee and Yu [5] adopt a structural approach borrowed from the literature of credit risk modeling in corporate finance (see Merton [7] for the first model) to value the reinsurance contract. They allow the reinsurer to transfer the risk to the capital market via CAT bonds and, in effect, to reduce the risk of the reinsurer's default risk. Since the payments from CAT bonds cannot be replicated by the ordinary types of securities available in financial markets, the pricing has to be done in the incomplete market model.

One common technique used for pricing in incomplete markets is indifference pricing via expected utility. Young [10] finds indifference prices under a stochastic interest rate for the case where investors receive unity if a catastrophe does not occur and 0 otherwise; see Young [10] for further references on indifference pricing. Our paper extends the result of [10] by incorporating a more complex payment structure. Namely, the CAT bond is issued in two tranches, specifically senior and junior tranches each with its own payment schedule. We price each tranche in the framework of "relative" indifference pricing. To our knowledge, this is the first paper that deals with a structured catastrophe derivative.

The remainder of the paper is organized as follows: In Section 2, we assume that reinsurance claims follow a (Poisson) jump-diffusion process, so the corresponding surplus process of the reinsurer also follows a jump-diffusion because we ignore investment earnings. We also describe the structured CAT bond that the reinsurer will issue in order to reduce its risk. In Section 3, we present our method for pricing the CAT bond; we rely on utility indifference pricing. In Section 4, we demonstrate our method with a number of examples while assuming that utility is exponential. First, we assume that the claims only follow a diffusion process; that is, we assume that there is no jump risk. Second, we assume that there is only a jump risk in the claim

\footnotetext{
${ }^{1}$ http://www.swissre.com/INTERNET/pwswpspr.nsf/
} 
process, with no diffusion in the claim process nor the index process. We end Section 4 with a numerical example in which there is both jump and diffusion risk in the claim process. Section 5 ends the paper.

\section{Problem Description}

Assume that a reinsurer faces a reinsurance contract with claim process

$$
C_{t}=a t-\sigma W_{t}+\sum_{i=0}^{N_{t}} Y_{i}, \quad C_{0}=0,
$$

in which $a$ and $\sigma$ are positive constants, and $Y_{1}, Y_{2}, \ldots$ are identically distributed positive random variables with common distribution $F(d y) . W$ is a Brownian motion, $N$ is a Poisson process with a constant arrival rate $\lambda$, and $N$ is independent of the $Y$ 's and of the Brownian motion $W$. The $Y$ 's represent infrequent claims, while we let the Brownian motion approximate the frequent claims.

The reinsurer receives insurance premiums continuously at the constant rate $(1+\theta)(a+\lambda \mathbb{E} Y)$ with a relative risk loading of $\theta>0$. We ignore investment earnings of the reinsurer. Therefore, the reinsurer's surplus process $X$ has state space $\mathcal{I}=\mathbb{R}$ with dynamics

$$
X_{t}=x_{0}+(1+\theta)(a+\lambda \mathbb{E} Y) t-a t+\sigma W_{t}-\sum_{i=0}^{N_{t}} Y_{i}=: x_{0}+\mu t+\sigma W_{t}-\sum_{i=0}^{N_{t}} Y_{i},
$$

in which

$$
\mu \triangleq(1+\theta) \lambda \mathbb{E} Y+\theta a
$$

and $x_{0}$ is the initial surplus assigned to this contract. Note that there is positive probability that the process $X$ hits the ruin state 0 . However, we assume that the reinsurer has a large capital (besides $x_{0}$ ) and this particular reinsurance contract takes up only a small percentage of the total wealth of the reinsurer. In other words, even if the process $X$ hits the zero state, the reinsurer has enough capital so that it does not fall into insolvency.

There are two ways for the reinsurer to reduce its risk: First, the reinsurer could accept proportionally less reinsurance with correspondingly proportionally less premium, so that the claim and surplus processes become, respectively,

$$
C_{t}^{\xi}=\xi C_{t}=\xi a t-\xi \sigma W_{t}+\xi \sum_{i=0}^{N_{t}} Y_{i}
$$

and

$$
X_{t}^{\xi}=x_{0}+\xi \mu t+\xi \sigma W_{t}-\xi \sum_{i=0}^{N_{t}} Y_{i}
$$

Alternatively, the reinsurer could issue a catastrophe risk bond, or CAT bond, to hedge possible large claims caused by catastrophic events. The purchasers of CAT bonds, in turn, receive amounts at the maturity time $T$, contingent upon the occurrence of these events. For example, Young [10] finds indifference prices under a stochastic interest rate for the case where investors receive unity if a catastrophe does not occur and 0 otherwise. 
In this paper, we handle the case where the CAT bonds are issued in two tranches, senior and junior tranches, to appeal to investors with different risk preferences. To simplify the argument, we assume that the two tranches are both zero-coupon bonds. The actual claims $C$ that the reinsurer experiences are measured against an index process $R$ whose dynamics are given by

$$
R_{t}=R_{0}+\rho t-\zeta W_{t}
$$

with known positive constants $\rho, \zeta$, and $R_{0}$. Note that for simplicity, we assume the Brownian motion in this index process is identical to the one in (2.1). We assume that $R$ is a standard against which the experience of the reinsurer can be measured. For example, it might represent the experience of all reinsurers in the same line of business as $C$. Alternatively, it could be a financial index that "smoothes" the experience $C$. Therefore, we assume that $\zeta \leq \sigma$; that is, $R$ 's diffusion is less volatile than $C$ 's.

To create a more realistic index, one could allow the diffusion in (2.6) to be correlated with the one in (2.1), instead of identical to it. Also, one could allow for jumps, perhaps by using the same Poisson process as in (2.1) with the jump size some fraction of $Y$. The differential equations in Section 3 below would retain the same form, although the details would change.

Define a stopping time, which we call the critical time, by

$$
\tau \triangleq \inf \left\{t>0: C_{t}>R_{t}\right\}
$$

to indicate the first time that the claim process $C$ becomes greater than the index process $R$. Note that the stopping time $\tau$ might be infinite, but this is not a problem for us because we consider a finite time horizon $T$.

To simplify the notation, we define an auxiliary process $Z$ by

$$
Z_{t} \triangleq C_{t}-R_{t}=z_{0}+(a-\rho) t-(\sigma-\zeta) W_{t}+\sum_{i=0}^{N_{t}} Y_{i},
$$

with $Z_{0}=C_{0}-R_{0}=-R_{0}=: z_{0}<0$. The critical time $\tau$ can be rewritten as $\tau=\inf \left\{t>0: Z_{t}>0\right\}$. See Rolski et al. [8] for references concerning the problem of ruin for a classical surplus process perturbed by a Brownian motion, as is the process $Z$.

If $\tau \leq T$, then the payoff to the junior tranche becomes zero, and the payoff to the senior tranche at time $T$ is a function of the overshoot $Z_{\tau}=C_{\tau}-R_{\tau}$. Recall that the claim process involves a compound Poisson process that may cause an overshooting (namely, $Z_{\tau}>0$ ). The larger the value of $Z_{\tau}$, the less the available funds for paying the investors of the CAT bonds; thus, we set the payout function to be a decreasing function of the overshoot. On the other hand, if $C$ does not hit $R$ (that is, $\tau>T$ ), then both senior and junior tranches receive 1 at time $T$ because there are more funds available to pay investors due to lower insurance claims. Specifically, we define the payoff function for the senior tranche by

$$
\begin{cases}c\left(Z_{\tau}\right), & \tau \leq T \\ 1, & \tau>T\end{cases}
$$


in which $c$ is a piecewise linear function of the form

$$
c(z)= \begin{cases}c_{1}-c_{2} \cdot z, & 0 \leq z \leq c_{1} / c_{2} \\ 0, & \text { otherwise }\end{cases}
$$

with $0<c_{1} \leq 1$ and $c_{2}>0$. The constants $c_{1}$ and $c_{2}$ control the riskiness of the senior tranche of CAT bond. The payoff to the junior tranche is given by

$$
\begin{cases}0, & \tau \leq T \\ 1, & \tau>T\end{cases}
$$

In the next section, we describe our scheme for pricing these junior and senior tranches via indifference pricing.

\section{Indifference Pricing}

We price this structured CAT bond via the following procedure. First assume that the reinsurer evaluates risk according to expected utility theory with an increasing, concave, smooth utility function $u: \mathbb{R} \rightarrow \mathbb{R}$.

0. As an auxiliary functional, consider

$$
W(x, t ; \xi) \triangleq \mathbb{E}\left[u\left(X_{T}^{\xi}\right) \mid X_{t}^{\xi}=x\right]
$$

in which $X^{\xi}$ is given by

$$
X_{T}^{\xi}=x+\xi \mu(T-t)+\xi \sigma\left(W_{T}-W_{t}\right)-\xi \sum_{i=N_{t}+1}^{N_{T}} Y_{i},
$$

and $\xi \in[0,1] . W$ is the utility of terminal wealth of the reinsurer if the reinsurer reduces its coverage to the proportion $\xi$. The function $W$ solves the partial differential equation (pde)

$$
\left\{\begin{array}{l}
W_{t}+\xi \mu W_{x}+\frac{1}{2} \xi^{2} \sigma^{2} W_{x x}-\lambda \int_{0}^{\infty}(W(x, t)-W(x-\xi y, t)) F(d y)=0 \\
W(x, T ; \xi)=u(x)
\end{array}\right.
$$

with the constant $\mu$ defined in (2.3).

1. In the absence of issuing CAT bonds, the reinsurer proportionally reduces its risk; that is, it chooses some number $\xi \in[0,1]$ to maximize its expected utility of terminal wealth. Specifically, it solves

$$
V^{j}(x, t) \triangleq \sup _{\xi \in[0,1]} W(x, t ; \xi) .
$$

Let $\xi^{*}$ denote the maximizer of the right-hand side of (3.3). Note that $\xi^{*}$ might depend on $x$ and $t$. 
2. Because a CAT bond has the effect of passing part of the reinsurer's exposure (to the catastrophe risk) to a third party (namely, the bond holders), an alternative way to reduce the exposure is to issue a CAT bond while fully reinsuring the cedent's claim. The expected utility of the seller of $\alpha>0$ units of the junior tranche of the CAT bond is

$$
U^{j}(x, z, t ; \alpha) \triangleq \mathbb{E}\left[u\left(X_{T}-\alpha \mathbf{1}_{\{\tau>T\}}\right) \mid X_{t}=x, Z_{t}=z, \max _{0 \leq s \leq t} Z_{s}<0\right] .
$$

Here, $X_{T}$ is given by (3.2) with $\xi=1$. The value function $U^{j}$ is the unique solution of the following integro-differential equation for $(x, z, t) \in \mathbb{R} \times \mathbb{R}_{-} \times[0, T]$, in which we suppress arguments,

$$
\left\{\begin{array}{l}
U_{t}^{j}+\mu U_{x}^{j}+(a-\rho) U_{z}^{j}+\frac{1}{2} \sigma^{2} U_{x x}^{j}-\sigma(\sigma-\zeta) U_{x z}^{j}+\frac{1}{2}(\sigma-\zeta)^{2} U_{z z}^{j} \\
\quad-\lambda \int_{0}^{-z}\left(U^{j}(x, z, t)-U^{j}(x-y, z+y, t)\right) F(d y) \\
\quad-\lambda \int_{-z}^{\infty}\left(U^{j}(x, z, t)-W(x-y, t)\right) F(d y)=0, \\
U^{j}(x, 0, t ; \alpha)=W(x, t), \\
U^{j}(x, z, T ; \alpha)=u(x-\alpha),
\end{array}\right.
$$

in which $W(x, t)=W(x, t ; \xi=1)$. The boundary condition $U^{j}(x, 0, t ; \alpha)=W(x, t)$ holds when $\sigma>0$ or $\zeta>0$. If $\sigma=0=\zeta$, then we have only one source of randomness, namely, the compound Poisson process, and we can reduce the dimensionality of the problem. See Section 4.2 for an example in which we consider this case.

3. The indifference price $h^{j}=h^{j}(x, z, t ; \alpha)$ of $\alpha$ units of the junior tranche solves

$$
V^{j}(x, t)=U^{j}\left(x+h^{j}, z, t ; \alpha\right) .
$$

We call $h^{j}$ the indifference price because the reinsurer is indifferent between reducing its risk by selling proportionally less reinsurance or by issuing $\alpha$ units of the junior tranche of the CAT bond and receiving $h^{j}$ in exchange.

4. To address more risk-averse investors, the reinsurer issues $\beta$ units of the senior tranche, additional to the junior tranche. The expected utility of the reinsurer in this case is

$$
U^{s}(x, z, t ; \alpha, \beta) \triangleq \mathbb{E}\left[u\left(X_{T}-\beta c\left(Z_{\tau}\right) \mathbf{1}_{\{\tau \leq T\}}-(\alpha+\beta) \mathbf{1}_{\{\tau>T\}}\right) \mid X_{t}=x, Z_{t}=z\right],
$$

in which the amount $c\left(Z_{\tau}\right)$ is paid at time $T$, with the function $c$ defined in (2.10) and with $Z$ following the dynamics in (2.8). As in (3.4), $X_{T}$ is given by (3.2) with $\xi=1$. For $(x, z, t) \in \mathbb{R} \times \mathbb{R}_{-} \times[0, T]$, one can show that $U^{s}$ uniquely solves

$$
\left\{\begin{array}{l}
U_{t}^{s}+\mu U_{x}^{s}+(a-\rho) U_{z}^{s}+\frac{1}{2} \sigma^{2} U_{x x}^{s}-\sigma(\sigma-\zeta) U_{x z}^{s}+\frac{1}{2}(\sigma-\zeta)^{2} U_{z z}^{s} \\
\quad-\lambda \int_{0}^{-z}\left(U^{s}(x, z, t)-U^{s}(x-y, z+y, t)\right) F(d y) \\
\quad-\lambda \int_{-z}^{c_{1} / c_{2}-z}\left(U^{s}(x, z, t)-W(x-y-\beta c(z+y), t)\right) F(d y) \\
\quad-\lambda \int_{c_{1} / c_{2}-z}^{\infty}\left(U^{s}(x, z, t)-W(x-y, t)\right) F(d y)=0, \\
U^{s}(x, 0, t ; \alpha, \beta)=W(x, t), \\
U^{s}(x, z, T ; \alpha, \beta)=u(x-(\alpha+\beta)) .
\end{array}\right.
$$


Again, the boundary condition $U^{s}(x, 0, t ; \alpha, \beta)=W(x, t)$ holds when $\sigma>0$ or $\zeta>0$.

5. Alternatively, instead of issuing the senior tranche, the reinsurer can reduce its reinsurance exposure proportionally, say, to $\pi \in[0,1]$ of the original risk $C$ on top of the already issued junior tranche. Expected utility in this case becomes

$$
J(x, z, t ; \alpha, \pi) \triangleq \mathbb{E}\left[u\left(X_{T}^{\pi}-\alpha \mathbf{1}_{\{\tau>T\}}\right) \mid X_{t}^{\pi}=x, Z_{t}=z\right],
$$

where $X_{T}^{\pi}$ is given in (3.2) with $\xi=\pi$. For $(x, z, t) \in \mathbb{R} \times \mathbb{R}_{-} \times[0, T], J$ uniquely solves

$$
\left\{\begin{array}{l}
J_{t}+\pi \mu J_{x}+(a-\rho) J_{z}+\frac{1}{2} \pi^{2} \sigma^{2} J_{x x}-\pi \sigma(\sigma-\zeta) J_{x z}+\frac{1}{2}(\sigma-\zeta)^{2} J_{z z} \\
\quad-\lambda \int_{0}^{-z / \pi}(J(x, z, t)-J(x-\pi y, z+\pi y, t)) F(d y) \\
\quad-\lambda \int_{-z / \pi}^{\infty}(J(x, z, t)-W(x-\pi y, t)) F(d y)=0, \\
J(x, 0, t ; \alpha, \pi)=W(x, t), \\
J(x, z, T ; \alpha, \pi)=u(x-\alpha) .
\end{array}\right.
$$

As before, the boundary condition $J(x, 0, t ; \alpha, \pi)=W(x, t)$ holds when $\sigma>0$ or $\zeta>0$. Then, we suppose the insurer finds the value of $\pi$ to maximize $J$. We define the corresponding value function $V^{s}$ by

$$
V^{s}(x, z, t ; \alpha) \triangleq \sup _{\pi \in[0,1]} J(x, z, t ; \alpha, \pi) .
$$

6. Finally, the relative indifference price $h^{s}=h^{s}(x, z, t ; \alpha, \beta)$ of $\beta$ units of the senior tranche of the CAT bond solves

$$
V^{s}\left(x+h^{j}, z, t ; \alpha\right)=U^{s}\left(x+h^{j}+h^{s}, z, t ; \alpha, \beta\right) .
$$

Recall that $h^{j}$ is calculated in the previous steps. We call $h^{s}$ a relative indifference price because it is the indifference price of the senior tranche in the presence of, or relative to, the junior tranche.

In the next section, we consider the case of exponential utility under three conditions. First, we assume that the only source of randomness is the diffusion; second, we assume that the only source of randomness is the compound Poisson process. Finally, we present a numerical example in which the claim process has both a diffusion and a compound Poisson component.

\section{Indifference Pricing Under Exponential Utility}

Throughout this section, we assume that the utility function $u$ is given by $u(x)=-e^{-\eta x}$ for some $\eta>0$. In Section 4.1, we assume that the only source of randomness is the diffusion. In this case, we find explicit solutions to $W, V^{j}, U^{j}, U^{s}$, and $V^{s}$; however, the indifference prices $h^{j}$ and $h^{s}$ are only available implicitly. For that reason, we also compute the indifference prices numerically for a specific example.

In Section 4.2, we assume that the only source of randomness is the compound Poisson process. In that case, we focus our computations on the case for which the jump size is constant. Finally, in Section 4.3, we give a numerical example in the presence of both a diffusion and a compound Poisson process. 


\subsection{Diffusion Only}

In this section, we assume that $\lambda=0$; that is, there is no source of randomness from the compound Poisson process. In this case, given $X_{t}^{\xi}=x$, we can determine the auxiliary process $Z$ from $x=x_{0}+\xi \mu t+\xi \sigma W_{t}$ as follows:

$$
\begin{aligned}
Z_{t} & =z_{0}+(a-\rho) t-(\sigma-\zeta) W_{t} \\
& =z_{0}+(a-\rho) t-(\sigma-\zeta)\left[\frac{x-x_{0}}{\xi \sigma}-\frac{\mu}{\sigma} t\right] \\
& =\left[z_{0}-(\sigma-\zeta) \frac{x-x_{0}}{\xi \sigma}\right]+\left[(a-\rho)+\frac{\mu}{\sigma}(\sigma-\zeta)\right] t .
\end{aligned}
$$

Therefore, we only need to consider the one state variable $X_{t}^{\xi}=x$, instead of both $X_{t}^{\xi}=x$ and $Z_{t}=z$.

The critical time $\tau$ occurs when $Z_{t}>0$ or when $x<\tilde{x}(t ; \xi)$, in which $\tilde{x}$ is defined by

$$
\tilde{x}(t ; \xi) \triangleq x_{0}+z_{0} \frac{\xi \sigma}{\sigma-\zeta}+\xi\left[(a-\rho) \frac{\sigma}{\sigma-\zeta}+\mu\right] t .
$$

When $x<\tilde{x}(t ; \xi)$ occurs (or actually when $x$ first equals $\tilde{x}(t ; \xi)$ because of the presence of the diffusion process), then the payoff to the junior tranche goes from $\alpha$ to 0 , and the payoff to the senior tranche goes from $\beta$ to $c_{1} \beta \leq \beta$.

In what follows, we determine the various functions given in the steps in Section 3 for this special case. After that, we present a numerical example in which we compute indifference prices for the junior and senior tranches.

0 . The value function when insuring proportion $\xi$ of the claims, namely $W$, is given by

$$
W(x, t ; \xi)=-\exp \left(-\eta x-\left(\xi \mu \eta-\frac{1}{2} \xi^{2} \sigma^{2} \eta^{2}\right)(T-t)\right) .
$$

1. The maximizer of $W$ is $\xi^{*}=1$ if $\frac{\mu}{\sigma^{2} \eta}>1$; otherwise, $\xi^{*}=\frac{\mu}{\sigma^{2} \eta}$. It follows that the value function under the optimal proportional amount to insure, namely $V^{j}$, is given by

$$
V^{j}(x, t)= \begin{cases}-\exp \left(-\eta x-\left(\mu \eta-\frac{1}{2} \sigma^{2} \eta^{2}\right)(T-t)\right), & \text { if } \frac{\mu}{\sigma^{2} \eta}>1 \\ -\exp \left(-\eta x-\frac{1}{2}\left(\frac{\mu}{\sigma}\right)^{2}(T-t)\right), & \text { otherwise. }\end{cases}
$$

2. The value function when issuing the junior tranche, namely $U^{j}$, solves the following pde:

$$
\left\{\begin{array}{l}
U_{t}^{j}+\mu U_{x}^{j}+\frac{1}{2} \sigma^{2} U_{x x}^{j}=0, \\
U^{j}(\tilde{x}(t), t ; \alpha)=W(\tilde{x}(t), t ; \xi=1)=-\exp \left(-\eta \tilde{x}(t)-\left(\mu \eta-\frac{1}{2} \sigma^{2} \eta^{2}\right)(T-t)\right), \\
U^{j}(x, T ; \alpha)=-e^{-\eta(x-\alpha)},
\end{array}\right.
$$

in which we write $\tilde{x}(t)$ for $\tilde{x}(t ; \xi=1)$. Under the assumption that

$$
\frac{a-\rho}{\sigma-\zeta}+\sigma \eta>0
$$


the solution of (4.5) is given by

$$
U^{j}(x, t ; \alpha)=-\exp \left(-\eta \tilde{x}(T)+\left(\eta \frac{\sigma(a-\rho)}{\sigma-\zeta}+\frac{1}{2} \sigma^{2} \eta^{2}\right)(T-t)\right) \tilde{U}^{j}(x, t ; \alpha),
$$

in which $\tilde{U}^{j}$ is given by

$$
\begin{aligned}
\tilde{U}^{j}(x, t ; \alpha)= & e^{-(x-\tilde{x}(t)) \eta}\left\{e^{\eta \alpha}+\left(1-e^{\eta \alpha}\right) \Phi\left(\sqrt{T-t}\left(\frac{a-\rho}{\sigma-\zeta}+\sigma \eta\right)-\frac{x-\tilde{x}(t)}{\sigma \sqrt{T-t}}\right)\right\} \\
& +e^{(x-\tilde{x}(t))\left(\frac{2(a-\rho)}{\sigma(\sigma-\zeta)}+\eta\right)}\left(1-e^{\eta \alpha}\right) \Phi\left(-\sqrt{T-t}\left(\frac{a-\rho}{\sigma-\zeta}+\sigma \eta\right)-\frac{x-\tilde{x}(t)}{\sigma \sqrt{T-t}}\right)
\end{aligned}
$$

with $\Phi$ the cumulative distribution function of the standard normal random variable. If the inequality (4.6) does not hold, then we have to replace some of the expressions in (4.7) by their absolute values, but the general form of $U^{j}$ will still be valid.

3. To solve $V^{j}(x, t)=U^{j}\left(x+h^{j}, t ; \alpha\right)$ for the indifference price of the junior tranche $h^{j}=h^{j}(x, t ; \alpha)$, we must use numerics due to the highly nonlinear nature of $x$ in the expression for $U^{j}$. We present a numerical example at the end of this subsection.

4. We can write the value function when issuing the senior tranche, namely $U^{s}$, in terms of $U^{j}$ from Step 2 as follows:

$$
\begin{aligned}
U^{s}(x, t ; \alpha, \beta) & =\mathbb{E}\left[u\left(X_{T}-\beta c_{1} \mathbf{1}_{\{\tau \leq T\}}-(\alpha+\beta) \mathbf{1}_{\{\tau>T\}}\right) \mid X_{t}=x\right] \\
& =e^{\eta \beta c_{1}} \mathbb{E}\left[-\exp \left\{-\eta\left(X_{T}-\left(\alpha+\beta\left(1-c_{1}\right)\right) \mathbf{1}_{\{\tau>T\}}\right)\right\} \mid X_{t}=x\right] \\
& =e^{\eta \beta c_{1}} U^{j}\left(x, t ; \alpha+\beta\left(1-c_{1}\right)\right) .
\end{aligned}
$$

5. The value function of the issuer of the junior tranche who reduces its reinsurance exposure proportionally to $\pi$, namely $J$, solves the following pde:

$$
\left\{\begin{array}{l}
J_{t}+\pi \mu J+\frac{1}{2} \pi^{2} \sigma^{2} J_{x x}=0, \\
J(\tilde{x}(t ; \pi), t)=W(\tilde{x}(t ; \pi), t ; \pi)=-\exp \left(-\eta \tilde{x}(t ; \pi)-\left(\pi \mu \eta-\frac{1}{2} \pi^{2} \sigma^{2} \eta^{2}\right)(T-t)\right), \\
J(x, T)=-e^{-\eta(x-\alpha)}
\end{array}\right.
$$

Under the assumption in (4.6), one can show that $J$ is given by

$$
J(x, t ; \alpha, \pi)=-\exp \left(-\eta \tilde{x}(T ; \pi)+\left(\pi \eta \frac{\sigma(a-\rho)}{\sigma-\zeta}+\frac{1}{2} \pi^{2} \sigma^{2} \eta^{2}\right)(T-t)\right) \tilde{J}(x, t ; \alpha, \pi),
$$

in which $\tilde{J}$ is given by

$$
\begin{aligned}
\tilde{J}(x, t ; \alpha, \pi)= & e^{-(x-\tilde{x}(t ; \pi)) \eta}\left\{e^{\eta \alpha}+\left(1-e^{\eta \alpha}\right) \Phi\left(\pi \sqrt{T-t}\left(\frac{a-\rho}{\sigma-\zeta}+\sigma \eta\right)-\frac{x-\tilde{x}(t ; \pi)}{\pi \sigma \sqrt{T-t}}\right)\right\} \\
& +e^{(x-\tilde{x}(t ; \pi))\left(\frac{2(a-\rho)}{\sigma(\sigma-\zeta)}+\eta\right)}\left(1-e^{\eta \alpha}\right) \Phi\left(-\pi \sqrt{T-t}\left(\frac{a-\rho}{\sigma-\zeta}+\sigma \eta\right)-\frac{x-\tilde{x}(t ; \pi)}{\pi \sigma \sqrt{T-t}}\right) .
\end{aligned}
$$

Then, one finds $\pi^{*} \in[0,1]$ to maximize $J$, and the corresponding value function is $V^{s}$. 
6. Finally, to solve $V^{s}\left(x+h^{j}, t ; \alpha\right)=U^{s}\left(x+h^{j}+h^{s}, t ; \alpha, \beta\right)$ for the relative indifference price of the senior tranche $h^{s}=h^{s}(x, t ; \alpha, \beta)$, we must use numerics due to the highly nonlinear nature of $x$ in the expression for $U^{s}$.

Often under exponential utility, indifference prices are independent of the value of the wealth (or surplus) process; see, for example, Young [10]. However, our prices of the CAT bonds depend on the value of the surplus process because the payment schedule of the tranches depends on surplus process $X$ via the claim process $C$ and how it relates to the index process $R$. For the same reason, as the risk parameter $\eta \rightarrow 0+$, the indifference prices cannot be express as expectations of the payouts of the CAT bonds. For example, one can show that as $\eta \rightarrow 0+$, the price for the junior tranche solves the following non-linear equation:

$$
\frac{1}{\alpha} \tilde{x}(t)=\Phi\left(\sqrt{T-t} \frac{a-\rho}{\sigma-\zeta}+\frac{x+h^{j}-\tilde{x}(t)}{\sigma \sqrt{T-t}}\right)-\Phi\left(\sqrt{T-t} \frac{a-\rho}{\sigma-\zeta}-\frac{x+h^{j}-\tilde{x}(t)}{\sigma \sqrt{T-t}}\right) .
$$

To end this section, we present a numerical example in which we compute indifference prices for the junior and senior tranches of a CAT bond when diffusion is the only source of randomness. The bond parameters are $\left(T, \alpha, \beta, c_{1}\right)=(10,1,1,0.3)$. For stochastic processes $C$ and $R$, we use $(a, \rho, \theta, \sigma, \zeta)=$ $(0.3,0.15,0.1667,0.275,0.225)$. For the exponential utility, $\eta=0.25$. In this case, it turns out that $\xi^{*}=1$ and $\pi^{*}=1$. Hence, the function $V^{s}$ identically equals $U^{j}$. The figures below show (a) the value functions $V^{j}, U^{j}=V^{s}$, and $U^{s}$, (b) the indifference price of junior tranche, $h^{j}$ and the relative indifference price of senior tranche, $h^{s}$, all as functions of the current surplus value, $x$. We evaluate the functions at $t=1$, with $x_{0}=2$ and $z_{0}=-2$. For the junior price, we observe that $X_{t}^{\xi}=x$ increases, $h^{j}(x, 1)$ increases and becomes flat at $h^{j}(x, 1)=1$. It is because $Z_{t}=z$ decreases when $x$ increases (see (4.1)) and accordingly the probability of CAT bond default gets smaller. However, $h^{j}(x, 1)$ is in essence insensitive to the value of $x$. In case of the senior price, it is effectively one, that is, $h^{s}(x, 1)=1$ for all $x>0$.

\subsection{Compound Poisson Process Only}

In this section, we assume that $\sigma=\zeta=0$; that is, there is no source of randomness from the diffusion. In this case, as in Section 4.1, given $X_{t}^{\xi}=x$, we can determine the auxiliary process $Z$ from $x=x_{0}+\xi \mu t-$ $\xi \sum_{i=1}^{N_{t}} Y_{i}$ as follows:

$$
Z_{t}=z_{0}+(a-\rho) t+\sum_{i=1}^{N_{t}} Y_{i}=z_{0}+(a-\rho) t+\left[\mu t-\frac{x-x_{0}}{\xi}\right]=\left[z_{0}-\frac{x-x_{0}}{\xi}\right]+(a-\rho+\mu) t .
$$

Therefore, we only need to consider the one state variable $X_{t}^{\xi}=x$, instead of both $X_{t}^{\xi}=x$ and $Z_{t}=z$. The critical time $\tau$ occurs when $Z_{t}>0$ or when $x<\bar{x}(t ; \xi)$, in which $\bar{x}$ is defined by

$$
\bar{x}(t ; \xi) \triangleq x_{0}+\xi\left(z_{0}+(a-\rho+\mu) t\right) .
$$

Additionally, in this section, we assume that the jumps are of a fixed size, that is, $F(d y)=\delta_{y} d y$ for some fixed $y \in \mathbb{R}_{+}$. 


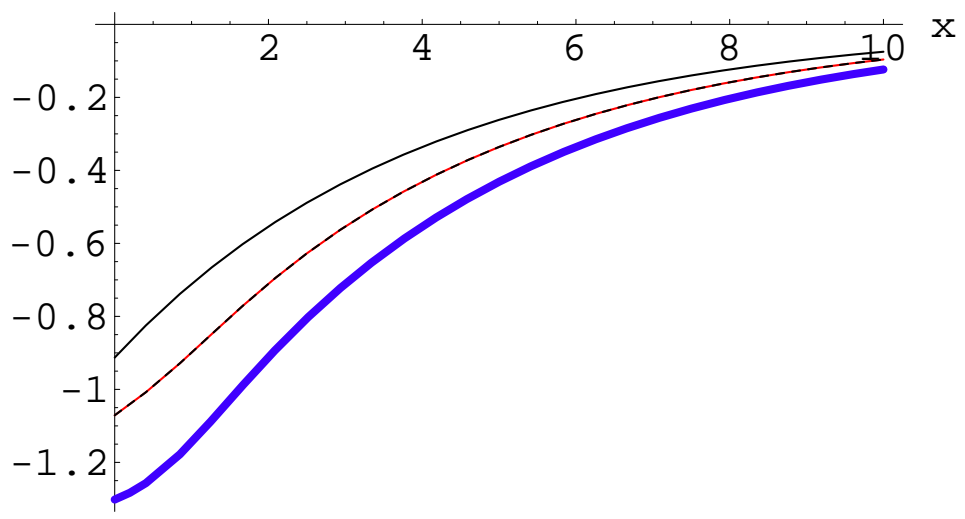

(a)

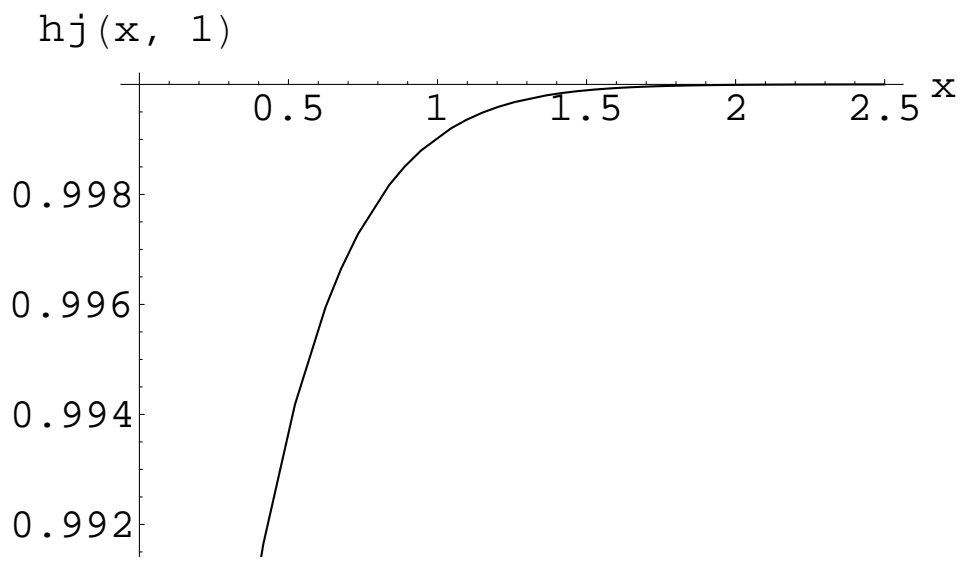

(b)

Figure 1: (a) the value functions $V^{j}$ (solid black), $V^{s}$ (dashed black), $U^{j}$ (red, same as $V_{s}$ ) and $U^{s}$ (bold purplle), (b) junior tranche price at $t=1, h^{j}(x, 1)$.

0 . The value function when insuring proportion $\xi$ of the claims, namely $W$, is given by

$$
W(x, t ; \xi)=-\exp (-\eta x+b(\xi)(T-t))
$$

where $b(\xi)$ is given by

$$
b(\xi)=-\xi \mu \eta+\lambda\left(e^{\xi \eta y}-1\right) .
$$

1. Next, we find $V^{j}(x, t)=\sup _{\xi \in[0,1]}-e^{-\eta x+b(\xi)(T-t)}$. The optimal proportion retained, namely $\xi^{*}$, is the value of $\xi$ that minimizes $b(\xi)$. Simple differentiation with respect to $\xi$ gives us $b^{\prime}(\xi)=-\mu \eta+$ $\lambda \eta y e^{\xi \eta y}$ with $b^{\prime \prime}(\xi)>0$ for all $\xi \in[0,1]$. Hence, there are three possible cases: (1) $\xi^{*}=0$ if $\ln \left(\frac{\mu}{\lambda y}\right) \leq 0$, (2) $\xi^{*}=\frac{1}{\eta y} \ln \left(\frac{\mu}{\lambda y}\right)$ if $0<\ln \left(\frac{\mu}{\lambda y}\right)<\eta y$, and (3) $\xi^{*}=1$ if $\ln \left(\frac{\mu}{\lambda y}\right) \geq \eta y$. The 
resulting $V^{j}$ is given by

$$
V^{j}(x, t)= \begin{cases}-e^{-\eta x}, & \text { if } \ln \left(\frac{\mu}{\lambda y}\right) \leq 0, \\ -e^{-\eta x+\left[\lambda\left(\frac{\mu}{\lambda y}-1\right)-\frac{\mu}{y} \ln \left(\frac{\mu}{\lambda y}\right)\right](T-t)}, & \text { if } 0<\ln \left(\frac{\mu}{\lambda y}\right)<\eta y, \\ -e^{-\eta x+\left(\lambda\left(e^{\eta y}-1\right)-\mu \eta\right)(T-t)}, & \text { if } \ln \left(\frac{\mu}{\lambda y}\right) \geq \eta y .\end{cases}
$$

2. The junior tranche pays zero if a jump of size $y$ takes $X_{t-}=x$ and moves it to $X_{t}=x-y<\bar{x}(t ; \xi=$ $1)=\left(x_{0}+z_{0}\right)+(a-\rho+\mu) t$, or equivalently,

$$
y>x-\left(x_{0}+z_{0}\right)-(a-\rho+\mu) t=x-\bar{x}(t)
$$

in which we write $\bar{x}(t)=\bar{x}(t ; \xi=1)$. Accordingly, for any jump distribution $F(d y)$, equations (3.4) and (3.5) become

$$
U^{j}(x, t ; \alpha)=\mathbb{E}\left[u\left(X_{T}-\alpha \mathbf{1}_{\{\tau>T\}}\right) \mid X_{t}=x, \max _{0 \leq s \leq t} X_{s} \geq \bar{x}(t ; \xi=1)\right]
$$

and

$$
\left\{\begin{array}{l}
U_{t}^{j}+\mu U_{x}^{j}-\lambda \int_{0}^{x-\bar{x}(t)}\left(U^{j}(x, t)-U^{j}(x-y, t)\right) F(d y) \\
\quad-\lambda \int_{x-\bar{x}(t)}^{\infty}\left(U^{j}(x, t)-W(x-y, t)\right) F(d y)=0, \\
U^{j}(x, T ; \alpha)=u(x-\alpha),
\end{array}\right.
$$

respectively. In the particular case of $F(d y)=\delta_{y} d y$, (4.21) becomes

$$
\left\{\begin{array}{l}
U_{t}^{j}+\mu U_{x}^{j}-\lambda\left(U^{j}(x, t)-\mathbf{1}_{\{y \leq x-\bar{x}(t)\}} U^{j}(x-y, t)-\mathbf{1}_{\{y>x-\bar{x}(t)\}} W(x-y, t)\right)=0, \\
U^{j}(x, T ; \alpha)=u(x-\alpha),
\end{array}\right.
$$

Since (4.22) does not allow an explicit solution, we resort to numerics to solve this equation.

3. To solve $V^{j}(x, t)=U^{j}\left(x+h^{j}, t ; \alpha\right)$ for the indifference price of the junior tranche $h^{j}=h^{j}(x, t ; \alpha)$, we must use numerics due to the nature of the equation for $U^{j}$, namely (4.21). We present a numerical example at the end of this subsection.

4. In the pure jump case for a jump distribution $F(d y)$, the value function when issuing the senior tranche, namely $U^{s}$, solves the following integro-differential equation:

$$
\left\{\begin{array}{l}
U_{t}^{s}+\mu U_{x}^{s}-\lambda \int_{0}^{x-\bar{x}(t)}\left(U^{s}(x, t)-U^{s}(x-y, t)\right) F(d y) \\
\quad-\lambda \int_{x-\bar{x}(t)}^{c_{1} / c_{2}+x-\bar{x}(t)}\left(U^{s}(x, t)-W(x-y-\beta c(-x+\bar{x}(t)+y), t)\right) F(d y) \\
\quad-\lambda \int_{c_{1} / c_{2}+x-\bar{x}(t)}^{\infty}\left(U^{s}(x, t)-W(x-y, t)\right) F(d y)=0, \\
U^{s}(x, T ; \alpha, \beta)=u(x-(\alpha+\beta)) .
\end{array}\right.
$$

In the particular case of $F(d y)=\delta_{y} d y$, this equation simplifies appropriately. 
5. The value function of the issuer of the junior tranche who reduces its reinsurance exposure proportionally to $\pi$, namely $J$, solves the following pde:

$$
\left\{\begin{array}{l}
J_{t}+\pi \mu J_{x}-\lambda \int_{0}^{(x-\bar{x}(t ; \pi)) / \pi}(J(x, t)-J(x-\pi y, t)) F(d y) \\
\quad-\lambda \int_{(x-\bar{x}(t ; \pi)) / \pi}^{\infty}(J(x, t)-W(x-\pi y, t)) F(d y)=0 \\
J(x, T ; \alpha, \pi)=u(x-\alpha) .
\end{array}\right.
$$

Then, one finds $\pi^{*} \in[0,1]$ to maximize $J$, and the corresponding value function is $V^{s}$.

6. Finally, to solve $V^{s}\left(x+h^{j}, t ; \alpha\right)=U^{s}\left(x+h^{j}+h^{s}, t ; \alpha, \beta\right)$ for the relative indifference price of the senior tranche $h^{s}=h^{s}(x, t ; \alpha, \beta)$, we must use numerics.

We present a numerical example in the next section in which both diffusion and jump components are incorporated.

\subsection{Diffusion and Compound Poisson Process}

In this section, we present a numerical example, in which we use the (numerical) model in Section 4.1 and add a jump component. Specifically, assume that the bond parameters are $\left(T, \alpha, \beta, c_{1}, c_{2}\right)=(10,1,1,0.3,1)$. For stochastic processes $C$ and $R$, we use $(a, \rho, \theta, \sigma, \zeta)=(0.3,0.15,0.1667,0.275,0.225)$. For the exponential utility, $\eta=0.25$. Note that the parameters up to this point are exactly the same as in the diffusiononly case. For this example, we evaluate at $t=0$ with $z_{0}=-4$ to obtain the initial prices $h^{j}\left(x, z_{0}, 0\right)$ and $h^{s}\left(x, z_{0}, 0\right)$ as function of initial surplus $X_{0}=x$. Here we write $X_{0}$ by $x$ to make it clear that our indifference prices are plotted as functions of the initial surplus level. At $t=0$, we can treat $x$ and $z$ independently for the purpose of looking into the pure effects of additional jump risk on the prices for a given value of the index process $R$, that is $z_{0}=-R_{0}=-4$. At this level, if there is no jump component $(\lambda=0)$, both junior and senior prices are $h^{j}\left(x, z_{0}, 0\right)=h^{s}\left(x, z_{0}, 0\right)=1$ for all $X_{0}=x>0$.

Now we add jump parameters $\lambda=0.02$ and constant jump size of $y=1$. In this case, it also turns out that $\xi^{*}=1$ and $\pi^{*}=1$. Hence the function $V^{s}$ identically equals $U^{j}$. The pictures in Figure 2 below at the last page are (a) the utility functions $V^{j}, V^{s}=U^{j}$ and $U^{s}$ and (b) the indifference prices $h^{j}\left(x, z_{0}, 0\right)$ and $h^{s}\left(x, z_{0}, 0\right)$. The horizontal distance between $V^{j}$ and $U^{j}$ is narrow, reflecting the lower junior price due to the significant increase of the probability of CAT bond default. Note that the prices are insensitive to the initial surplus level, a similar observation to the case of diffusion only. Owing to the more favored payoff to the senior tranche, the price is not significantly affected. We observe how the additional risk component of the jump process significantly influences the price of the junior tranche in this example. This explains one of the reasons for the reinsurance company issuing distinct tranches in accordance with the investor's risk-reward preference.

\section{Summary}

We present a method for pricing structured CAT bonds based on utility indifference pricing, as in Young [10]. Our contributions to the literature of CAT bond pricing are two-fold. First, we apply indifference 
pricing to structured CAT bonds. We find a price for the senior tranche as a relative indifference price, that is, relative to the price of the junior tranche. Alternatively, one could take the approach that the senior tranche is priced first and the price of the junior tranche is relative to that. Second, instead of simply supposing that the "not-issue-a-CAT-bond" strategy of the reinsurer is to do nothing, we suppose that the reinsurer reduces its risk by reinsuring proportionally less of the claims, as modeled by the value functions $V^{j}$ and $V^{s}$ in this paper.

For future research in this area, we encourage the interested reader to consider a more realistic index process in (2.6). As we mentioned earlier, one could allow the diffusion in (2.6) to be correlated with the one in (2.1), instead of identical to it. Also, one could allow for jumps, perhaps by using the same Poisson process as in (2.1) with the jump size some fraction of $Y$. Additional numerical work would prove useful. For example, if the jump size $Y$ has a heavy tail, then the payoff of the senior tranche will match that of the junior tranche much of the time, so we expect their prices to be close. Conversely, if the jump size $Y$ has a light tail, as in our example in Section 4.3, then we expect the price of the senior tranche to be greater than that of the junior tranche, as we observed in our example.

\section{References}

[1] S. Cox and H. Pedersen. Catastrophe risk bonds. North American Actuarial Journal, 48:56-82, 2000.

[2] A. Dassios and J. Jang. Pricing of catastrophe reinsurance and derivatives using the cox process with shot noise intensity. Finance Stochast., 7:73-95, 2003.

[3] H. Geman and M. Yor. Stochastic time changes in catastrophe option pricing. Insurance: Mathematics and Economics, 21:185-193, 1997.

[4] S. Jaimungal and T. Wang. Catastrophe options with stochastic interest rates and compound poisson losses. Insurance: Mathematics and Economics, 38:469-483, 2005.

[5] J. Lee and M. Yu. Variation of catastrophe reinsurance with catastrophe bonds. Insurance: Mathematics and Economics, 2007 (to appear).

[6] C. McGhee. Market Update: The catastrophe bond market at year-end 2003. Guy Carpenter and Company, Inc. and MMC Security Corporation, 2004.

[7] R. Merton. On the pricing of corporate debt: The risk structure of interest rates. Journal of Finance, 29 (2):449$470,1974$.

[8] T. Rolski, H. Schmidli, V. Schmidt, and J. Teugels. Stochastic Processes for Insurance and Finance. Wiley, New York, 1999.

[9] P. Shimpi. Integrating Corporate Risk Management. Texere, New York, 2001.

[10] V. R. Young. Pricing in an incomplete market with an affine term structure. Mathematical Finance, 14(3):359$381,2004$. 


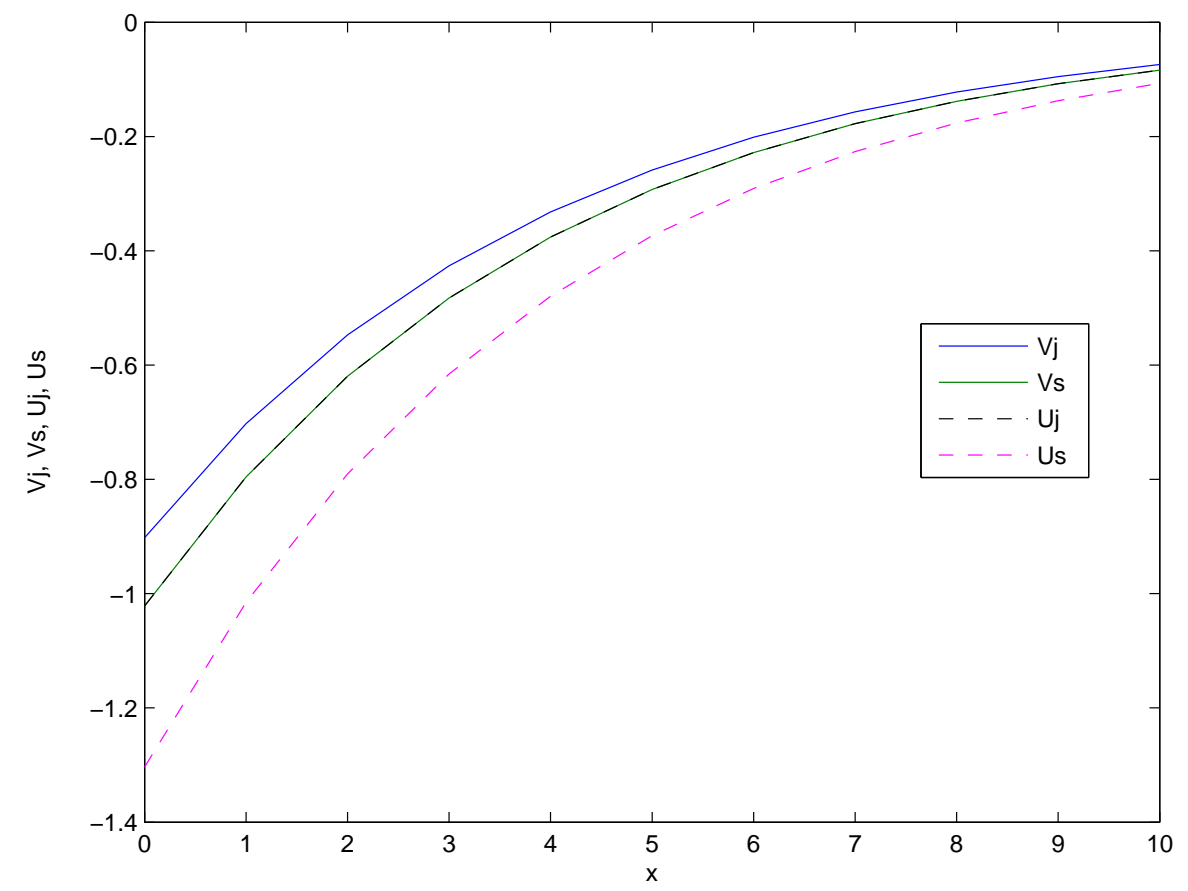

(a)

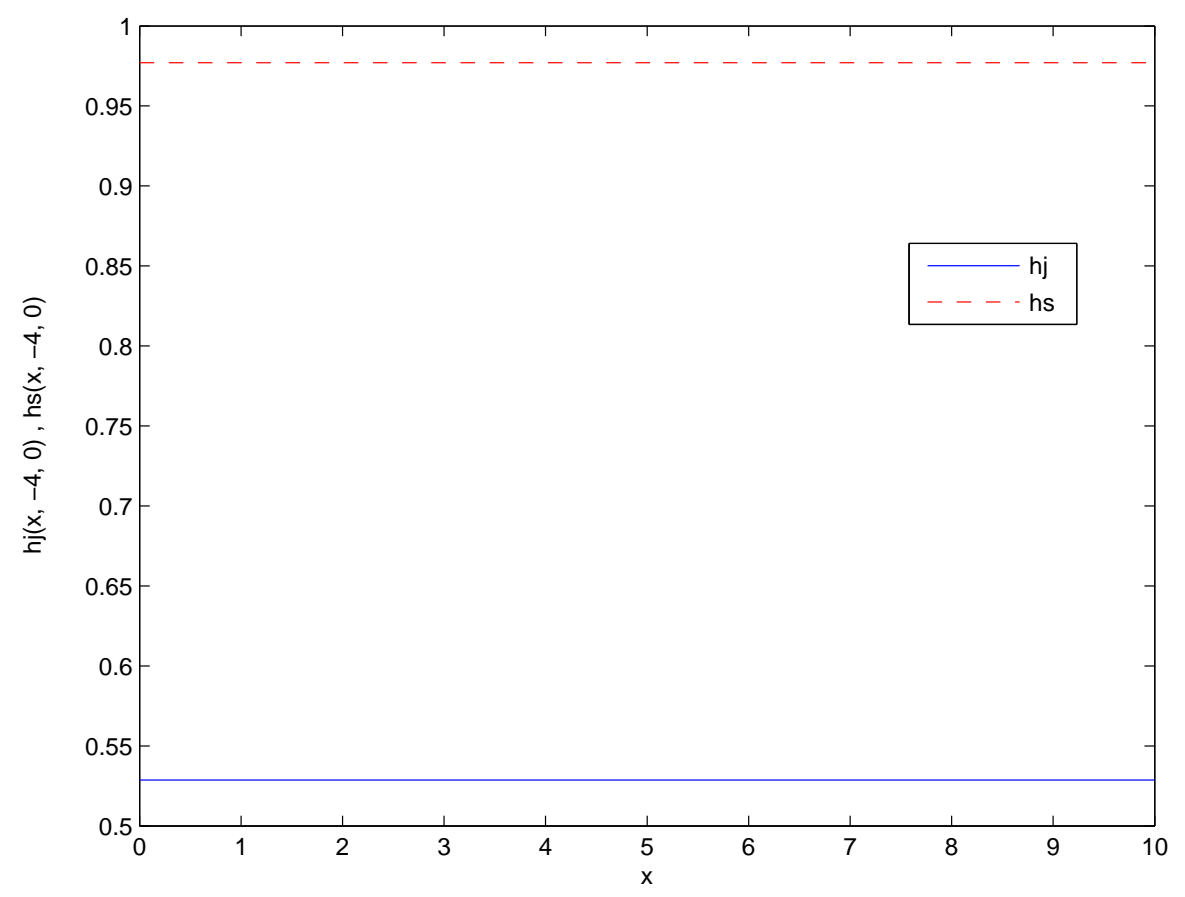

(b)

Figure 2: Jump-diffusion case: (a) the value functions $V^{j}$ (solid blue), $V^{s}$ (solid green, same as $U^{j}$ and overridden), $U^{j}$ (solid black) and $U^{s}$ (dashed magneta), (b) junior tranche price at $t=0, h^{j}(x,-4,0)$ (solid blue), and senior tranche price, $h^{s}(x,-4,0)$ (dashed red). 